\title{
The conformal anomaly in N-dimensional spaces having a hyperbolic spatial section
}

\author{
A.A. Bytsenko \\ Department of Theoretical Physics, State Technical University \\ St Petersburg 195251, Russia \\ E. Elizalde 円 \\ Center for Advanced Study CEAB, CSIC, Camí de Santa Bàrbara, 17300 Blanes \\ and Department ECM and IFAE, Faculty of Physics, University of Barcelona, \\ Diagonal 647, 08028 Barcelona, Catalonia, Spain \\ and \\ S.D. Odintsov \\ Department ECM, Faculty of Physics, University of Barcelona, \\ Diagonal 647, 08028 Barcelona, Catalonia, Spain
}

\begin{abstract}
The conformal anomaly for spinors and scalars on a $N$-dimensional hyperbolic space is calculated explicitly, by using zeta-function regularization techniques and the Selberg trace formula. In the case of conformally invariant spinors and scalars the results are very much related with those corresponding to a $N$-dimensional sphere.
\end{abstract}

PACS: 04.62.+v, 04.60.-m, 02.30.+g

\footnotetext{
${ }^{1}$ E-mail: eli@zeta.ecm.ub.es

${ }^{2}$ E-mail: odintsov@ecm.ub.es. On leave from: Tomsk Pedagogical Institute, 634041 Tomsk, Russia.
} 
The conformal anomaly [1] plays an important role in different physical situations. Among others one can mention string theory [2], the $C$-theorem and its generalizations [3], and the anomaly-induced effective action [4]. Recently, an effective theory of quantum gravity has been constructed on the basis of anomaly-induced dynamics [5]. Moreover, the conformal anomaly (as well as the total energy-momentum tensor) can be used for the description of particle production in a gravitational field (see [6] for a review). The general structure of the conformal anomaly in $N$ dimensions has been studied in Ref. [7]. However, the explicit calculation of the conformal anomaly in a general, curved spacetime is actually possible only for $N=2,4,6$ or 8 . Already in the last case, $N=8$, the calculation is extremely involved.

Under such unfavourable circumstances, in order to obtain some information about the conformal anomaly in higher dimension a possible way is to consider the conformal anomaly in some specified background. Thus, for instance, the calculation of the conformal anomaly on a $N$-dimensional sphere $(N$ even) has been carried out in Ref. [8] using $\zeta$-function regularization [9] (see [10] for a review and detailed list of references). It has been pointed out in [8] that the conformal anomaly on a $N$-dimensional sphere changes sign as $(-1)^{1+N / 2}$.

The purpose of this paper is to study the structure of the conformal anomaly on $\mathrm{N}$ dimensional even hyperbolic spaces. One is led to apply the technique of the Selberg trace formula in order to calculate the corresponding zeta function. In this way we will obtain a remarkably simple expression for the conformal anomaly on such a space.

As is well known, for constant conformal transformations the variation of the connected vacuum functional $W(W=-\ln Z$, where $Z$ is the partition function) can be expressed in terms of the generalized $\zeta$-function [9] associated with the Laplace-Beltrami operator $L_{N}$ [1]

$$
\delta W=-\zeta\left(0 \mid L_{N}\right) \ln \mu^{2}=\frac{1}{2} \int d V<T_{\mu \nu}(x)>\delta g^{\mu \nu}(x),
$$

where $g^{\mu \nu}(x)$ is the metric, $\mu$ is a renormalization mass parameter and $<T_{\mu \nu}(x)>$ means that all connected vacuum graphs of the stress-energy tensor $T_{\mu \nu}(x)$ are to be included. Then Eq. (1) leads to

$$
<T_{\mu}^{\mu}(x)>= \pm V_{N}^{-1} \zeta\left(0 \mid L_{N}^{ \pm}\right),
$$

where for $S^{N}: V_{N}=2 \pi^{(N+1) / 2} a^{N} / \Gamma((N+1) / 2)$, while for the compact manifold $H^{N} / \Gamma$, with $\Gamma$ a co-compact group of discrete isometries: $V_{N}=V\left(\mathcal{F}_{N}\right) a^{N}$, a being the radius of the compact space and $V\left(\mathcal{F}_{N}\right)$ the volume of the fundamental domain $\mathcal{F}_{N}$. The sign + (resp. - ) corresponds to integer spin- $s$ fields (resp. spinors). 
We will consider the heat-kernel and $\zeta$-function, respectively, related with the LaplaceBeltrami operator $L_{N}=-\nabla^{\mu} \nabla_{\mu}$ acting on fields in $H^{N} / \Gamma$, with strictly hyperbolic group $\Gamma$. The elliptic operator $L_{N}$ has a pure discrete spectrum with isolated eigenvalues $\lambda_{j}$, $j=0,1,2, \ldots$, of finite multiplicity. With all these assumptions we shall now apply the Selberg trace formula to the scalar field [1]. It is convenient to distinguish between the contribution coming from the identity and the hyperbolic elements of the isometry group $\Gamma$. Thus, for the case of a scalar field $(s=0)$, we have

$$
\begin{aligned}
\operatorname{Tr} \exp \left(-t L_{N}^{+}\right)= & V\left(\mathcal{F}_{N}\right) e^{-t b^{2}} \int_{0}^{\infty} d r e^{-t r^{2}} \Phi_{N}(r)+\frac{e^{-t b^{2}}}{(4 \pi t)^{1 / 2}} \sum_{\{\gamma\}} \sum_{n=1}^{\infty} \frac{\chi^{n}(\gamma) l_{\gamma}}{S_{N}\left(n ; l_{\gamma}\right)} \exp \left[-\frac{\left(n l_{\gamma}\right)^{2}}{4 t}\right] \\
& \equiv K\left(t \mid L_{N}^{+}\right)+K_{h}\left(t \mid L_{N}^{+}\right)
\end{aligned}
$$

and

$$
\begin{aligned}
\zeta_{H^{N} / \Gamma}\left(z \mid L_{N}^{+}\right)= & V\left(\mathcal{F}_{N}\right) \int_{0}^{\infty} d r\left(r^{2}+b^{2}\right)^{-z} \Phi_{N}(r)+\frac{1}{\sqrt{\pi} \Gamma(z)} \sum_{\{\gamma\}} \sum_{n=1}^{\infty} \frac{\chi^{n}(\gamma) l_{\gamma}}{S_{N}\left(n ; l_{\gamma}\right)}\left(\frac{2 b}{n l_{\gamma}}\right)^{1 / 2-z} \\
& \times K_{1 / 2-z}\left(n l_{\gamma} b\right) \equiv \zeta\left(z \mid L_{N}^{+}\right)+\zeta_{h}\left(z \mid L_{N}^{+}\right)
\end{aligned}
$$

where $K_{\nu}(z)$ is the modified Bessel function of the second kind, $\{\gamma\}$ denotes the primitive conjugacy class determined by the element $\gamma \in \Gamma, l_{\gamma}$ is the length of the closed geodesic $\gamma$ (which is the same for all elements in the class $\{\gamma\}$ ), $\chi(\gamma): \Gamma \rightarrow S^{1}$ is a character of $\Gamma$, $S_{N}\left(n ; l_{\gamma}\right)$ some function of $n$ and $l_{\gamma}$ (see [11] for more details), and where we have set $a=1$ (in the final results the dependence on the radius $a$ will be easily restored). Moreover,

$$
\Phi_{N}(r)=C_{N} \mu^{+}(r, s=0), \quad C_{N}=2^{N-3} \pi^{-(N / 2+1)} \Gamma(N / 2),
$$

where $\mu^{+}(r, s)$ is the Plancherel measure [12].

We will now consider the generalization of these formulas to the case of symmetric transverse and traceless tensor fields and to spinors. In addition, the Selberg trace formula associated with rank-s eigentensors of the Laplace-Beltrami operator can be written in the same form (3), (4), in the appropriate space. For rank-1 eigenvectors, for example, the character $\chi$ of $\Gamma$ acts in a complex linear space $V$. Therefore, the second term in Eqs. (3) and (14) must be generalized in the sense that $\chi^{n}(\gamma) \rightarrow \operatorname{Tr}_{V}\left(\chi^{n}(\gamma)\right)$, where $\operatorname{Tr}_{V}$ denotes the trace operator in the corresponding space $V$ [13]. Notice, however, that these second terms do not contribute to the heat-kernel expansion (expansion for $t \rightarrow 0$ ), nor to the $\zeta$-function at $z=0$. So we will concentrate our interest on the functions $K\left(t \mid L_{N}\right)$ and $\zeta\left(z \mid L_{N}\right)$ only. 
In what follows we will evaluate the trace anomaly for spin-s fields $(s=0,1,2, \ldots)$ and Dirac spinors in anti-DeSitter spacetimes of even dimension $N$. For $N \geq 2$, we have [14]

$$
\begin{aligned}
& \mu^{ \pm}(r, s)=\frac{\pi r}{\left[2^{N-2} \Gamma(N / 2)\right]^{2}} \tanh [\pi(r+i s)] \sigma^{ \pm}(r, s) \\
& \tanh [\pi(r+i s)]= \begin{cases}\tanh (\pi r), & s=0,1, \ldots \\
\operatorname{coth}(\pi r), & s=1 / 2,3 / 2, \ldots\end{cases}
\end{aligned}
$$

where

$$
\begin{gathered}
\sigma^{+}(r, s)=\left[r^{2}+\left(s+\frac{N-3}{2}\right)^{2}\right] \prod_{j=1 / 2}^{(N-5) / 2}\left(r^{2}+j^{2}\right) \equiv \sum_{k=0}^{N / 2-1} \beta_{k, N}^{+} r^{2 k}, \\
\sigma^{-}(r, 1 / 2)=\prod_{j=1}^{N / 2-1}\left(r^{2}+j^{2}\right) \equiv \sum_{k=0}^{N / 2-1} \beta_{k, N}^{-} r^{2 k},
\end{gathered}
$$

the coeficients $\beta_{k, N}^{ \pm}$being defined by the recasting of the products into polynomials in $r^{2}$, in Eqs. (7) and (8). For $N=4$ the productory in Eq. (7) is omitted and we have: $\beta_{1,4}^{+}=1$, $\beta_{0,4}^{+}=(s+1 / 2)^{2}$ and $\beta_{0,2}^{-}=1$. The spectral functions on $H^{2}$ for spin 0 and 1 are both given by $\mu^{+}(r, 0)=\pi r \tanh (\pi r)$.

Substituting (6)-(8) into (3) and (44), we obtain

$$
\begin{gathered}
K\left(t \mid L_{N}^{+}\right)=g(s) A(N) e^{-t b^{2}} \int_{0}^{\infty} d r \sum_{k=0}^{N / 2-1} \beta_{k, N}^{+} e^{-t r^{2}} r^{2 k+1} \tanh (\pi r), \\
K\left(t \mid L_{N}^{-}\right)=2^{N / 2} A(N) e^{-t c^{2}} \int_{0}^{\infty} d r \sum_{k=0}^{N / 2-1} \beta_{k, N}^{-} e^{-t r^{2}} r^{2 k+1} \operatorname{coth}(\pi r), \\
\zeta\left(z \mid L_{N}^{+}\right)=g(s) A(N) \int_{0}^{\infty} d r \sum_{k=0}^{N / 2-1} \beta_{k, N}^{+} \frac{r^{2 k+1} \tanh (\pi r)}{\left(r^{2}+b^{2}\right)^{z}}
\end{gathered}
$$

and

$$
\zeta\left(z \mid L_{N}^{-}\right)=2^{N / 2} A(N) \int_{0}^{\infty} d r \sum_{k=0}^{N / 2-1} \beta_{k, N}^{-} \frac{r^{2 k+1} \operatorname{coth}(\pi r)}{\left(r^{2}+c^{2}\right)^{z}}
$$

where

$$
A(N)=\frac{V\left(\mathcal{F}_{N}\right)}{2^{N-1} \pi^{N / 2} \Gamma(N / 2)}, \quad g(s)=\frac{(2 s+N-3)(s+N-4) !}{(N-3) ! s !}, \quad N \geq 4 .
$$

Using the identities

$$
\begin{gathered}
\tanh [\pi(r+i s)]=1-\frac{2}{1+\exp [2 \pi(r+i s)]}, \quad \int_{0}^{\infty} \frac{d r r^{2 n-1}}{e^{2 \pi r}+1}=\frac{(-1)^{n-1}\left(1-2^{1-2 n}\right)}{4 n} B_{2 n} \\
\int_{0}^{\infty} \frac{d r r^{2 n-1}}{e^{2 \pi r}-1}=\frac{(-1)^{n-1}}{4 n} B_{2 n}
\end{gathered}
$$


where the $B_{n}$ are Bernoulli's numbers, we obtain

$$
\begin{aligned}
& K\left(t \mid L_{N}^{+}\right)=\frac{1}{2} g(s) A(N) e^{-t b^{2}} \sum_{k=0}^{N / 2-1} \beta_{k, N}^{+}\left[k ! t^{-k-1}+(-1)^{k+1} \sum_{l=0}^{\infty} \frac{t^{l}}{l !} \frac{1-2^{-2 k-2 l-1}}{k+l+1} B_{2 k+2 l+2}\right], \\
& K\left(t \mid L_{N}^{-}\right)=2^{N / 2-1} A(N) e^{-t c^{2}} \sum_{k=0}^{N / 2-1} \beta_{k, N}^{-}\left[k ! t^{-k-1}+(-1)^{k} \sum_{l=0}^{\infty} \frac{t^{l}}{l !(k+l+1)} B_{2 k+2 l+2}\right] .
\end{aligned}
$$

The integrals in Eqs. (11) and (12) converge for Re $z>N / 2$ and may be analytically continued in the complex $z$-plane by using the identities (14). As a result, we obtain (see also [10])

$$
\begin{aligned}
& \zeta\left(z \mid L_{N}^{+}\right)=\frac{1}{2} g(s) A(N) \sum_{k=0}^{N / 2-1} \beta_{k, N}^{+}\left[b^{2 k-2 z+2} B(k+1, z-k-1)-4 \int_{0}^{\infty} \frac{d r r^{2 k+1}}{\left(r^{2}+b^{2}\right)^{z}\left(e^{2 \pi r}+1\right)}\right] \\
& \zeta\left(z \mid L_{N}^{-}\right)=2^{N / 2-1} A(N) \sum_{k=0}^{N / 2-1} \beta_{k, N}^{-}\left[c^{2 k-2 z+2} B(k+1, z-k-1)+4 \int_{0}^{\infty} \frac{d r r^{2 k+1}}{\left(r^{2}+c^{2}\right)^{z}\left(e^{2 \pi r}-1\right)}\right],
\end{aligned}
$$

in terms of the beta function $B(x, y)=\Gamma(x) \Gamma(y) / \Gamma(x+y)$. The last terms in Eqs. (17) and (18) are analytic in $z$ while the first ones give simple poles for $\zeta\left(z \mid L_{N}^{ \pm}\right)$at $z=1, \ldots, N / 2$, in agreement with the general theory [15]. For further evaluations in terms of Laurent series, we may rewrite the analytic terms in a more useful form, taking into account the Mellin transform of the factor $\exp (2 \pi r) \pm 1$ :

$$
[\exp (2 \pi r) \pm 1]^{-1}=\frac{1}{2 \pi i} \int_{\omega-i \infty}^{\omega+i \infty} d \sigma \zeta^{ \pm}(\sigma) \Gamma(\sigma)(2 \pi r)^{-\sigma}
$$

where $\operatorname{Re} \sigma=\omega, \omega>0$ (resp. $\omega>1$ ) for integer (resp. half-integer) $s, \zeta^{-}(\sigma)=\zeta(\sigma)$ is the Riemann $\zeta$-function, and $\zeta^{+}(\sigma)=\left(1-2^{1-\sigma}\right) \zeta(\sigma)=\sum_{n=1}^{\infty}(-1)^{n-1} n^{-\sigma}=\eta(\sigma)$, for $\operatorname{Re} \sigma>0$, the $\eta$-function.

Using (19) in the last terms of (17) and (18) and performing the integration over $r$ with the help of the beta function $B(x, y)$ (notice that, owing to absolute convergence, the order of integration over $r$ and $\sigma$ can be interchanged), we obtain

$$
\begin{aligned}
\zeta\left(z \mid L_{N}^{+}\right)= & \frac{g(s) A(N)}{2 \Gamma(z)} \sum_{k=0}^{N / 2-1} \beta_{k, N}^{+} b^{2 k-2 z+2}[\Gamma(k+1) \Gamma(z-k-1) \\
& \left.-\frac{1}{\pi i} \int_{\omega-i \infty}^{\omega+i \infty} d \sigma \zeta^{+}(\sigma) \Gamma(\sigma) \Gamma(k+1-\sigma / 2) \Gamma(z-k-1+\sigma / 2)(2 \pi b)^{-\sigma}\right]
\end{aligned}
$$

and

$$
\begin{aligned}
\zeta\left(z \mid L_{N}^{-}\right)= & \frac{2^{N / 2-1} A(N)}{\Gamma(z)} \sum_{k=0}^{N / 2-1} \beta_{k, N}^{-} c^{2 k-2 z+2}[\Gamma(k+1) \Gamma(z-k-1) \\
& \left.+\frac{1}{\pi i} \int_{\omega-i \infty}^{\omega+i \infty} d \sigma \zeta^{-}(\sigma) \Gamma(\sigma) \Gamma(k+1-\sigma / 2) \Gamma(z-k-1+\sigma / 2)(2 \pi c)^{-\sigma}\right] .
\end{aligned}
$$


The analytic continuation of the spin- $\zeta$-function at $z=0$ can be obtained by making use of the asymptotic expansion of $\Gamma(z)$ at $z=-m, m \in \mathbf{N}$ 16]

$$
\begin{aligned}
\Gamma(z)= & \frac{(-1)^{m}}{m !}\left\{(z+m)^{-1}+\psi(m+1)+\frac{z+m}{2}\left[\frac{\pi^{2}}{3}+\psi^{2}(m+1)-\psi^{\prime}(m+1)\right]\right. \\
& \left.+\mathcal{O}\left((z+m)^{2}\right)\right\}
\end{aligned}
$$

where $\psi(z)=d \ln \Gamma(z) / d z$. Thus, we get

$$
\zeta\left(0 \mid L_{N}^{+}\right)=\frac{1}{2} g(s) A(N) \sum_{k=0}^{N / 2-1} \frac{(-1)^{k+1}}{k+1} \beta_{k, N}^{+}\left[b^{2 k+2}+\left(1-2^{-2 k-1}\right) B_{2 k+2}\right]
$$

and

$$
\zeta\left(0 \mid L_{N}^{-}\right)=2^{n / 2-1} A(N) \sum_{k=0}^{N / 2-1} \frac{(-1)^{k+1}}{k+1} \beta_{k, N}^{-}\left[c^{2 k+2}-B_{2 k+2}\right] .
$$

Restoring now the dependence on the radius $a$, we obtain the final result

$$
\begin{gathered}
<T_{\mu}^{\mu}(x)>_{+}=\frac{g(s)}{(4 \pi)^{N / 2} \Gamma(N / 2) a^{N}} \sum_{k=0}^{N / 2-1} \frac{(-1)^{k+1}}{k+1} \beta_{k, N}^{+}\left[b^{2 k+2}+\left(1-2^{-2 k-1}\right) B_{2 k+2}\right], \\
<T_{\mu}^{\mu}(x)>_{-}=\frac{1}{(2 \pi)^{N / 2} \Gamma(N / 2) a^{N}} \sum_{k=0}^{N / 2-1} \frac{(-1)^{k+1}}{k+1} \beta_{k, N}^{-}\left[B_{2 k+2}-c^{2 k+2}\right] .
\end{gathered}
$$

Of the two expressions, let us analyze first the one corresponding to a spinor field. Notice that in the case of a compact manifold, the $\zeta$-function corresponding to a spinor field is well defined, since the spectrum of the Dirac operator does not include the zero point. For massless spinors $c=0$, and we have

$$
<T_{\mu}^{\mu}(x)>_{-}=\frac{1}{(2 \pi)^{N / 2} \Gamma(N / 2) a^{N}} \sum_{k=0}^{N / 2-1} \frac{(-1)^{k+1}}{k+1} B_{2 k+2} \beta_{k, N}^{-} .
$$

Therefore, in accordance with [8], for massless spinors we obtain

$$
<T_{\mu}^{\mu}\left(x \in H^{N}\right)>_{-}=(-1)^{N / 2}<T_{\mu}^{\mu}\left(x \in S^{N}\right)>_{-}
$$

and, for $N=2,<T_{\mu}^{\mu}(x)>_{-}=-\left(12 \pi a^{2}\right)^{-1}$. As we see, there appears a very simple relation (only the sign can be different) between the conformal anomaly for a spinor on a sphere and on a hyperbolic space.

For the minimally coupled scalar field of mass $m, b^{2}=\rho_{N}^{2}+a^{2} m^{2}$, where $\rho_{N}=(N-1) / 2$. For the conformally invariant scalar field in $N$ dimensions, we have

$$
b^{2}=\rho_{N}^{2}+\frac{(N-2) a^{2}}{4(N-1)} R(x)
$$




\begin{tabular}{|c|c|c|c|}
\hline \hline$N$ & $<T_{\mu}^{\mu}(x)>_{c}^{h y p}$ & $<T_{\mu}^{\mu}(x)>_{c}^{s p h}$ & numerical \\
\hline \hline 4 & $-\frac{1}{240 \pi^{2}}$ & $-\frac{1}{240 \pi^{2}}$ & $=-0.00042217$ \\
\hline 6 & $-\frac{5}{4032 \pi^{3}}$ & $\frac{5}{4032 \pi^{3}}$ & $=0.00003999$ \\
\hline 8 & $-\frac{23}{34560 \pi^{4}}$ & $-\frac{23}{34560 \pi^{4}}$ & $=-6.83211 \cdot 10^{-6}$ \\
\hline 10 & $-\frac{263}{560880 \pi^{5}}$ & $\frac{263}{560880 \pi^{5}}$ & $=1.53227 \cdot 10^{-6}$ \\
\hline \hline
\end{tabular}

Table 1: Values of the scalar conformal anomaly on hyperbolic spaces of dimension $N=4,6,8,10$, respectively (the radius $a$ has been set equal to 1 ). The results are compared with the corresponding ones for the case of a sphere, which are given in the last two columns. We see that the only difference is a sign when $N / 2$ is odd.

where $R(x)=-N(N-1) / a^{2}$ is the scalar curvature. As a consequence, $b^{2}=1 / 4$ and

$$
<T_{\mu}^{\mu}(x)>_{c}=\frac{1}{(4 \pi)^{N / 2} \Gamma(N / 2) a^{N}} \sum_{k=0}^{N / 2-1} \frac{(-1)^{k+1}}{k+1} \beta_{k, N}^{+}\left[2^{-4 k-2}+\left(1-2^{-2 k-1}\right) B_{2 k+2}\right] .
$$

In particular,

$$
<T_{\mu}^{\mu}(x)>_{c}^{N=2}=-\frac{1}{12 \pi a^{2}}, \quad<T_{\mu}^{\mu}(x)>_{c}^{N=4}=-\frac{1}{240 \pi^{2} a^{4}}
$$

where we have used, for $N=2, \beta_{0,2}^{+}=1, g(0)=1$. The values of the conformal anomaly in the scalar case, for several dimensions $N=4,6,8,10$ (and $a=1$ ) are given in Table 1 . They are again the same as the corresponding ones in spherical space, but for a factor $(-1)^{N / 2}$, as in the spinor case.

We should draw attention to the fact that, in the case of the spin-1, vector field theory, for instance, the Hodge-de Rahm operator $-(d \delta+\delta d)$ acting on co-exact one-forms is associated with the massless operator $\left[-\nabla^{\mu} \nabla_{\mu}+(N-1) a^{-2}\right] g_{\mu \nu}$. The eigenvalues of this operator are $r^{2}+\left(\rho_{N}-1\right)^{2}$ [14], and for the Proca field of mass $m$ we find $b^{2}=\left(\rho_{N}-1\right)^{2}+a^{2} m^{2}$.

In conclusion, we have evaluated the conformal anomaly in spacetimes of arbitrary dimension which possess a compact spatial section of the form $H^{N} / \Gamma$. We have restricted ourselves to the situation where the manifold is smooth and $\Gamma$ is a discrete subgroup of $\mathrm{SO}(\mathrm{N}, 1)$, acting freely and properly discontinously on $H^{N}$. In this case, the 'topological terms' on the right hand side of Eqs. (3) and (4) do not contribute to the heat kernel expansion, as we have seen above. This has simplified the calculations somehow, nevertheless we have also discussed shortly more general situations. The Selberg trace formula for the case 
when the group $\Gamma$ contains elliptic elements (orbifolds) - together with the corresponding $\zeta$-function and the heat-kernel coefficients - can be found in [11]. Extension of the above procedure to the explicit evaluation of the conformal anomaly in such case seems certainly feasible.

\section{Acknowledgments}

SDO would like to thank the members of the Department ECM, Barcelona University, for continued hospitality. This work has been supported by DGICYT (Spain), project No. PB93-0035, by CIRIT (Generalitat de Catalunya), and by RFFR (Russia), project No. 9402-03234. 


\section{References}

[1] S. Deser, M. Duff and C.J. Isham, Nucl. Phys. B111 (1976) 45; L.S. Brown and J.P. Cassidy, Phys. Rev. D15 (1977) 2810; for a review, see N. Birrell and P.C.W. Davies, Quantum Fields in Curved Space-Time (Cambridge Univ. Press, Cambridge, 1982).

[2] A.M. Polyakov, Phys. Lett. B103 (1981) 207.

[3] A.B. Zamolodchikov, JETP Lett. 43 (1986) 730; J.L. Cardy, Phys. Lett. B215 (1988) 749; I. Jack and H. Osborn, Nucl. Phys. B343 (1990) 647; A. Capelli, D. Friedan and J.I. Latorre, Nucl. Phys. B352 (1991) 616.

[4] R. Reigert, Phys. Lett. B134 (1984) 56; I.L. Buchbinder, S.D. Odintsov and I.L. Shapiro, Phys. Lett. B162 (1985) 93; E.S. Fradkin and A.A. Tseytlin, Phys. Lett. B134 (1984) 187; E.T. Tomboulis, Nucl. Phys. B329 (1990) 410; S.D. Odintsov and I.L. Shapiro, Class. Quant. Grav. 8 (1991) L57; J.S. Dowker, preprint MUTP-14 (1994)

[5] I. Antoniadis and E. Mottola, Phys. Rev. D46 (1992) 2013; S.D. Odintsov, Z. Phys. C54 (1992) 531; I. Antoniadis, P.O. Mazur and E. Mottola, Nucl. Phys. B388 (1992) 627; I. Antoniadis and S.D. Odintsov, Mod. Phys. Lett. A8 (1993) 979; E. Elizalde and S.D. Odintsov, Phys. Lett. B315 (1993) 245.

[6] L. Parker, in Recent Developments in Gravitation, M. Levy and S. Deser Eds. (Plenum, New York, 1979).

[7] S. Deser and A. Schwimmer, Phys. Lett. B309 (1993) 279.

[8] E. Copeland and D. Toms, Class. Quant. Grav. 3 (1986) 431.

[9] S.W. Hawking. Commun. Math. Phys., 55 (1977) 133; J.S. Dowker and R. Critchley. Phys. Rev. D13 (1976) 224.

[10] E. Elizalde, S.D. Odintsov, A. Romeo, A.A. Bytsenko and S. Zerbini, Zeta regularization techniques with applications (World Sci., Singapore, 1994).

[11] Chavel, Eigenvalues in Riemannian Geometry (Academic Press, USA, 1984); A.A. Bytsenko and S. Zerbini, Class. Quant. Grav. 9 (1992) 1365; A.A. Bytsenko, G. Cognola, L. Vanzo and S. Zerbini, preprint U.T.F. 325 (1994); A.A. Bytsenko, G. Cognola and L. Vanzo, J. Math. Phys. 33 (1992) 3108; erratum: 34 (1993) 1614. 
[12] S. Helgason, Groups and Geometrical Analysis (Academic Press, New York, 1984).

[13] A. B. Venkov, Proc. Steklov Inst. Math. 4 (1982) 1.

[14] R. Camporesi and A. Higuchi, Phys. Rev. D47 (1993); J. Math. Phys. 35 (1994) 4217; R. Camporesi, Commun. Math. Phys. 148 (1992) 283.

[15] S. Minakshisundaram and A. Pleijel, Can. J. Math. 1 (1949); for details see also R. Camporesi, Phys. REp. 196 (1990) 1.

[16] A. Erdélyi, Ed., Higher Transcendental Functions, Vol. I (McGraw-Hill, New York, 1953). 